\title{
Brain diffusion imaging and tractography to distinguish clinical severity of human PLP1-related disorders
}

\section{Authors}

Catherine Sarret ${ }^{1,2^{*}}$, Jean-Jacques Lemaire ${ }^{1,3}$, Anna Sontheimer ${ }^{1,3}$, Jérôme Coste ${ }^{1,3}$, Nadia Savy ${ }^{1,2}$, Bruno Pereira $^{4}$, Bastien Roche ${ }^{5}$, Odile Boespflug-Tanguy ${ }^{5,6}$.

${ }^{1}$ Institut Pascal, CNRS, Université Clermont Auvergne, SIGMA, France

${ }^{2}$ Service de génétique médicale, Centre de compétence des leucodystrophies et leucoencéphalopathies de cause rare, CHU Clermont-Ferrand, France

${ }^{3}$ Service de Neurochirurgie, CHU Clermont-Ferrand, France

${ }^{4}$ Unité de Biostatistiques (Délégation Recherche Clinique \& Innovation), CHU Clermont-Ferrand, France

${ }^{5}$ Service de Neurologie Pédiatrique et Maladies Métaboliques, Centre de référence des leucodystrophies et leucoencéphalopathies de cause rare, CHU-APHP Robert-Debré, Paris, France.

${ }^{6}$ INSERM UMR1141 PROTECT Université Paris-Diderot-Sorbonne Paris Cité, France

*Correspondence: Catherine Sarret, Equipe IGCNC (Institut Pascal, UMR CNRS-UCA-SIGMA), CHU de Clermont-Ferrand, France, 58 rue Montalembert, 63003 Clermont-Ferrand Cedex, France. Tel: +33(0)473750296. Fax: +33(0)473751201. E-mail: csarret@ chu-clermontferrand.fr. 


\begin{abstract}
Aims: We performed quantitative diffusion tensor imaging and brain tractography to distinguish clinical severity in a series of 35 patients with hypomyelinating PLPl-related disorders classified using the Motor Developmental Score according to best motor function acquired before 5 years and the gross motor function measure (GMFM) at the time of MRI acquisition.

Methods: We calculated fractional anisotropy and diffusivity values in 26 regions of interest, and the numbers of fibers and volumes of hemisphere tractograms. Fiber bundles on tractograms were characterized according to three criteria: size, direction of main-stream fibers, and connectivity of bundles (extratelencephalic projections, commissural fibers, and intrahemispheric connections).

Results: Age-adjusted multivariate analysis in three severity groups revealed increased isotropic diffusion in the superior cerebellar peduncle and grey matter in the most severe group, and larger tractogram volumes and increased numbers of fibers in the least severe group. Tractogram patterns showed preserved extratelencephalic projections and a main anterior-posterior aspect of intrahemispheric fibers in most patients, whereas interhemispheric connectivity was variable. The most severe and intermediate patients had less intrahemispheric connectivity, with a frequent predominant anterior-posterior direction of main-stream fibers.

Interpretation: Diffusion tensor imaging and tractographic parameters can operate as biomarkers to distinguish clinical severity in PLPl-related disorders and could improve our understanding of hypomyelinating leukodystrophies.
\end{abstract}

\title{
Key-words
}

PLP1-related disorders, Pelizaeus-Merzbacher disease, Spastic paraplegia type 2, diffusion tensor imaging, tractography, Proteolipid Protein

Running Title: DTI in PLPI-related disorders 


\section{Introduction}

Pelizaeus-Merzbacher disease (PMD, OMIM312080) and spastic paraplegia type 2 (SPG2, OMIM312920) are X-linked hypomyelinating leukodystrophies caused by mutations in the Proteolipid protein 1 (PLP1) gene that encodes the major central nervous system (CNS) myelin proteins, PLP and DM20 in oligodendrocytes [1,2]. The clinical findings associated with PLP1 mutations span a broad-spectrum continuum extending from severe congenital PMD forms to relatively mild late-onset SPG2, leading to the concept of PLP1-related disorders [3,4]. We previously classified patients according to the best motor function achieved between the ages of 2 and 5 years to demonstrate a genotype-phenotype correlation [3]. PMD1 (head control) and PMD2 (sitting position) are the most frequent forms and are usually associated with PLPI duplications. Missense mutations are rare but usually cause the most severe forms without motor acquisitions and dystonia (PMD0, also named "congenital" forms). Myelin deficiency is the main histological feature in human and mouse models of these severe forms [5,6]. PLP1 loss-of-function (null mutations and large deletions) leads to the mildest PMD3/SPG2 forms with acquisition of walking capacities with/without aid and relatively preserved cognitive development. Murine models and humans lacking PLP1 are characterized by axonal degeneration with a small enlargement of the interlamellar space of the myelin sheath $[2,7]$. In all forms, the myelin deficiency is demonstrated by the extensive abnormalities of multimodal evoked potentials and the diffuse hypomyelinated pattern of the supratentorial white matter (WM) magnetic resonance imaging (MRI) [8]. Most patients clinically improve during childhood, exactly like patients with cerebral palsy. Some progression of myelination is observed until 12 years of age [9]. The second decade is marked by neurological deterioration with cortico-subcortical atrophy on MRI, leading to severe quadriplegia, amyotrophy, optic atrophy and cognitive decline in young adults, even in the mildest forms [10]. Several recent studies have underlined the correlation between clinical severity and brain atrophy on MRI $[9,11,12]$.

Using diffusion tensor imaging (DTI), Laukka et al. found that the WM mean and radial diffusivities of 12 PLPl-mutated patients were higher than controls, in accordance with the severe myelin deficiency observed in PMD [13]. Axial diffusivity, which is assumed to be more specific to axon properties, was much less affected, except in the most severe patients. Here we investigated the ability of DTI parameters to distinguish the different severity groups (PMD0-1, PMD2, and PMD34) and to correlate with clinical severity in a cohort of 35 PLP1-mutated patients. We extended the WM analysis with tractography in an attempt to identify patterns of WM fiber damage. 


\section{Patients and methods}

\section{Patients}

Thirty-five patients from 32 families with a genetically-confirmed diagnosis of PLP1 mutation (19 duplications, 9 missense mutations and 7 null mutations) were included in this prospective study from January 2004 to December 2009, after first obtaining approval from the institutional review board (CPP Sud-Est VI No. AU788, CNIL No. 1406552, AFSSAPS No. B90298-60) and signed informed consent from the parents. For MRI acquisition, sedation by intrarectal pentobarbital: 3 to 5 $\mathrm{mg} / \mathrm{kg}$ (maximum dose: $100 \mathrm{mg}$ ) and oral alimemazine (4\% drops, 1 drop/kg, i.e. $1.25 \mathrm{mg} / \mathrm{kg}$ ), was performed in patients aged under 6 years old or requiring sedation to avoid movement artifacts.

Our study focused on a comparison of patients with distinct degrees of disease severity. Patients were classified according to the best motor developmental score (MDS) observed between age 2 and 5 years: PMD0, without motor acquisition ( $n=4$ patients); PMD1, head control ( $n=5)$; PMD2, sitting position ( $n=16)$; PMD3, walking with support ( $n=8)$; PMD4, independent walking ( $n=2)$. An 88-item gross motor function measure (GMFM) was assessed for all patients at the time of MRI acquisition for motor clinical severity [14]. MDS, GMFM, genotype and ages at GMFM and MRI acquisitions are summarized in Table 1. At the first MRI, median age was 8.6 years (2.3 to 43.5). Quantitative DTI measures were obtained in 32 out of 35 patients at median age 8.6 years (2.3 to 41.0). Tractography was carried on in 24 out of 35 patients at median age 13.6 years (2.3-43.5). For some patients, we could not analyze quantitative DTI $(n=3)$ or tractography $(n=11)$ maps due to corrupted data after MRI acquisition. Data were considered corrupted when DTI and tractography software could not read DICOM series because of artefacts due to subject motion. For eight patients, the quantitative DTI and tractography were analyzed on two different MRI scans at different ages in order to obtain non-corrupted data.

\section{Diffusion imaging and parameters}

Images were acquired on a 1.5-Tesla Siemens MR scanner under sedation. DTI acquisition used a single-shot echo-planar dual spin-echo sequence: repetition time (TR) $=3600 \mathrm{~ms}$, echo time (TE) $=85 \mathrm{~ms}, 90^{\circ}$ flip angle; six diffusion gradients; $\mathrm{b} 0=600 \mathrm{~s} / \mathrm{mm}^{2} ; 35$ joined axial slices; $256 \times 256$ matrix; voxel size $=2 \times 2 \times 6 \mathrm{~mm}^{3}$; total acquisition time $=6 \mathrm{~min}$. T1-weighted, T2-weighted and fluidattenuated inversion recovery (FLAIR) sequences were added for anatomical guidance (see Supplementary Material 1 for acquisition details). 
The DTI parameters fractional anisotropy (FA), mean diffusivity (MD), axial diffusivity (AD) and radial diffusivity (RD) were computed with DTI studio (Jiang and Mori, Johns Hopkins University, cmrm.med.jhmi.edu or godzilla.kennedykrieger.org) using a multivariate linear fitting algorithm for diffusion tensor calculation [15]. We explored 26 regions of interest (ROI), 17 in supratentorial and infratentorial WM and 9 in cortical and deep grey matter (GM) (listed in Supplementary Material 2), after automatic correction of eddy current distortion and automatic segmentation of ROI on the diffusion data based on Harvard-Oxford cortical and subcortical structural atlases (FSL, FMRIB Software Library, fsl.fmrib.ox.ac.uk).

\section{Tractograms}

Tractography was computed after correction of eddy current distortion, co-registration (mutual information algorithm) and anterior commissure - posterior commissure alignment. Right and left tractograms were generated by a trained neurologist (CS), using fiber assignment by continuous tracking (FACT) [16] and tensor deflection (TEND) [17] methods implemented within iPlan Stereotaxy 3.0 software (BrainLab, Feldkirchen, Germany). FA threshold was set at 0.20 with minimum fiber length tracking of $40 \mathrm{~mm}$. For each tractogram, volume and number of fibers (NOF) were computed and volume/ total intracranial volume (TIV) and NOF/TIV ratios were calculated. TIV was preferred over brain volume for normalization in order to consider brain growth in children and progressive atrophy in adults. The tractograms were interpreted at individual level and visually compared against two control subjects (aged of 8 and 41 years) by two experienced neurologists (CS and JJL) using the following morphological criteria of fibers bundles: (1) size: large, small, lacking; (2) direction of main-stream fibers (DMSF): anterior-posterior (longitudinal), lateral-medial (transversal), superior-inferior (vertical); (3) connectivity, irrespective of direction, e.g. fugal or petal: extratelencephalic projections (ETP), commissural fibers (CF) and intrahemispheric connections (IHC).

\section{Statistical analyses}

Statistical analysis was performed using Stata 13 software (Stata-Corp, College Station, TX). The tests were two-sided, with a type-I error set at $\alpha=0.05$. Quantitative data, expressed as mean and standard deviation or median and min-max ages according to statistical distribution, were compared between three independent groups (PMD0-1, PMD2, and PMD3-4) using a Kruskal-Wallis test followed by the appropriate post-hoc Dunn's test. ANCOVA was performed with age as the adjustment covariate. The assumption of the normality of residuals was also studied using the Shapiro-Wilk test. Data exhibiting non-normal distributions, a log-transformation was proposed to achieve normality of several variables and to allow the correct use of these analyses. We chose to 
report all the individual $p$-values without doing any mathematical correction for distinct tests comparing groups [18]. A focus was given to the magnitude of improvement and to the clinical relevance [19]. Results were expressed as effect-sizes and 95\% confidence intervals, and can be compared to the effect-size bounds proposed by Cohen [20], i.e. small $( \pm 0.2)$, medium $( \pm 0.5)$ and large ( \pm 0.8 , "grossly perceptible and therefore large"). Finally, a nonparametric Spearman's correlation test, with Sidak's type-I error correction, was used to compare individual quantitative DTI parameters and GMFM.

\section{Results}

\section{Patient severity}

The best motor developmental score (MDS) of the 32 patients analyzed with quantitative DTI were classified as follows: PMD0-1 $(n=8)$, PMD2 $(n=14)$ and PMD3-4 $(n=10)$, with median ages of 8.6 years (3.5-17.4), 6.0 years (2.3-19.1) and 15.4 years (5.5-41.0), respectively. Tractography analysis were possible in 3 PMD0-1 (median ages of 8.5 years (3.0-17.4)), 12 PMD2 (median ages 7.7 years (2.3-43.5)) and 9 PMD3-4 (median age 17.4 years (10.8-37.0)) patients.

The level of the motor handicap at the time of the MRI acquisition was evaluated in the 32 patients using the gross motor function measure (GMFM). Comparison of mean GMFM adjusted to the age between the three MDS groups showed that GMFM was higher in PMD3-4 than PMD0-1 and PMD2. It was also higher in PMD2 than PMD0-1 but not significantly (Fig. 1).

\section{Quantitative diffusion parameters}

Results of FA, MD, AD, RD in 17 ROI of WM and 9 ROI of GM are shown in Figure 2 (see Supplementary Table 1 for values and Supplementary Fig. 1 for effect-sizes and 95\% confidence intervals). We found that FA was significantly lower in PMD2 than PMD3-4 in middle cerebellar peduncle, in PMD0-1 than PMD2 in middle temporal gyrus, right caudate nucleus and right thalamus, and in PMD0-1 than PMD3-4 in postcentral gyrus. MD was significantly higher in PMD01 than PMD2 in superior cerebellar peduncle, frontal pole and left caudate nucleus, and in PMD0-1 than PMD3-4 in superior cerebellar peduncle. AD was significantly higher in PMD0-1 than PMD2 in superior cerebellar peduncle, left posterior and right superior corona radiata, frontal pole and left caudate nucleus, and in PMD0-1 than PMD3-4 in left superior cerebellar peduncle and right superior corona radiata. RD was significantly higher in PMD0-1 than PMD2 in superior cerebellar peduncle, frontal pole, middle temporal gyrus, caudate nuclei and thalami, and in PMD0-1 than PMD3-4 in right superior cerebellar peduncle and right caudate nucleus. 
Motor abilities, evaluated by the GMFM performed at the time of MRI, showed a relatively strong negative linear correlation with $\mathrm{MD}(p<0.043)$ and $\mathrm{AD}(p<0.036)$ in the superior cerebellar peduncles. Other possible strong linear relations were observed with $\mathrm{MD}, \mathrm{AD}$ or RD in anterior corona radiata but were not statistically significant (Fig. 3; Supplementary Table 2).

\section{Tractograms}

Volume/TIV and NOF/TIV ratios were higher in PMD3-4 than in other groups $(p<0.02)$ after adjustment to age, considering an older age of PMD3-4 patients at MRI acquisition (Fig. 4, Supplementary Table 3). ETP appeared relatively preserved in all groups. CF were variably individualized in all groups, ranging from large fibers $(n=9)$ to small fibers $(n=8)$ or a lack of connectivity $(n=7)$. IHC size was clinical severity-dependent, being clearly smaller $(n=8)$ or lacking connectivity (n=4) in PMD2 and PMD0-1 patients. DMSF of IHC was analyzable in 9 out of 9 PMD3-4 patients and 10 out of 12 PMD2 patients, and analysis showed major tracking of longitudinal fibers in 18 out of 19 patients whereas one patient had major transversal fibers (Fig.5A; Supplementary Fig.2 and 3). There were age-dependent differences in the PMD2 group, with smaller $\mathrm{CF}$ and IHC in the youngest and oldest patients while patients in late childhood had the largest projections (Fig.5B; Supplementary Fig.2 and 3).

\section{Discussion}

Here we analyzed quantitative DTI measures and tractography patterns from acquisitions performed at the same medical center in a large cohort of PLP1-mutated male patients in order to investigate the ability of these technics to distinguish severity groups. Our 35 patients were representative of the broad clinical spectrum observed in PLP1-related disorders: 46\% classical PMD2 forms, 26\% severe PMD0-1 forms, and 28\% mild PMD3-SPG2 forms. For clinical classification, we used the MDS previously demonstrated to correlate to genotype [3]. To correlate motor skills at the time of MRI, we used GMFM [14], a validated clinical score in cerebral palsy that is widely used in the quantification of motor abilities in leukodystrophic patients. Comparison of mean GMFM between MDS groups argued as in CP for a correlation between the level of the best motor development always observed before 5 years of age and the severity of the motor disabilities later in life.

This study shows that quantitative DTI parameters in supratentorial WM are insufficient to distinguish clinical severity in PLP1-mutated patients, whereas increased isotropic diffusion in cortical and deep GM and in infratentorial WM was clinical severity-linked. We also found clinical severity-dependent differences on quantitative and descriptive tractography. 
Laukka et al. previously identified increased MD and RD and decreased FA in supratentorial WM regions in 12 PMD patients compared to controls as expected in hypomyelinating brains [13]. Steenweg et al. also found that RD in supratentorial WM was inversely correlated with motor handicap in a cohort of 28 patients with various hypomyelinating disorders [21]. Here, comparison of the MDS groups of our PLPl-mutated patients failed to differentiate clinical severity using quantitative DTI parameters in supratentorial WM. A previous study in the same cohort of patients was unable to classify clinical severity using a myelination score on conventional MRI [9]. Moreover, motor abilities at the time of MRI using GMFM did not significantly decrease with increasing diffusion in supratentorial WM. These results confirmed that increased diffusion and decreased myelination score on the supratentorial WM demonstrate the myelin defect but may not serve to classify the clinical severity observed in PLPl-related disorders. Factors that may have limited the ability of this study to identify differences include the small sample sizes in each group, the broad age-ranges in the patients, or the algorithms and tools used in the quantitative analyses. Other factors could be an offset between microarchitectural changes and clinical features. Progression in both motor acquisitions and myelination are observed in the first decade of life but followed by motor and cognitive decline and progressive cerebral atrophy [9], so microarchitectural changes in supratentorial WM may precede clinical changes, creating a time gap between diffusion data and clinical data.

In infratentorial WM, clinical severity of the MDS groups and motor abilities at the time of MRI are in accordance with the DTI parameter values, except FA. This result was surprising, as infratentorial WM is usually well myelinated on structural MRI in PLPl-related disorders regardless of clinical severity [19]. However, Laukka et al. also found changes in MD and RD in cerebellar peduncle of PMD patients compared to controls [13]. Moreover, infratentorial abnormalities had been described in a DTI study by Hassen et al. that found MD, AD and RD variations in spinal cord of a SPG2 mouse model [22]. These results suggest that PLP1-mutated patients present subtle changes in infratentorial WM microarchitecture. This idea is consistent with (i) the early and predominant signs of cerebellar dysfunctions observed in PMD patients, (ii) the correlation we had previously found between clinical severity and cerebellar atrophy [9], and (iii) neuropathological analysis of PMD patients with cerebellar abnormalities [23]. In this study, we found higher AD and RD in the most severe patients. Laukka et al. also found changes in $\mathrm{AD}$, which is supposed to reflect axonal integrity, in only the most severe patients, whereas RD, which may reflect myelin integrity, was increased in all forms [13]. 
In cortical and deep GM, clinical severity of MDS groups increased with the increase of diffusion (MD, AD and RD) in several regions. Moreover, diffusion was more isotropic (decreased FA) in GM in most severe patients. To date in PLP1-related disorders, there are no reports of DTI analysis of GM but Laukka et al. and our team already showed a clear cerebral atrophy including GM compartment and correlating with clinical severity $[9,13]$. Hassen et al. also found MD increase in GM of spinal cord in a SPG2 mouse model [22]. Increased diffusion in cerebral GM has been reported in multiple sclerosis, where it appears to correlate to motor and cognitive decline [24,25], and in an animal model of genetic demyelinating disorder [26]. However, contrary to our patients, isotropic diffusion decreased (increased FA) in the cortex in multiple sclerosis as a probably result of local activation and proliferation of microglia characterized by the loss of ramifications and acquiring a more anisotropic, bipolar oriented structure as observed in active acute and chronic cortical lesions [25]. In PLP1-related disorders, it may corroborate with (i) the neuron loss, demyelination and axonal damage reported on neuropathological analysis of PLP1-mutated animal models [27] and PMD/SPG2 patients [23], (ii) the absence of local microglial activation and (iii) the neuronal PLP1 expression found in mouse and humans [28,29]. These results underline that GM is clearly involved in the severity of $P L P 1$-related disorders.

Tractography was possible in PLPl-related disorders, including severe forms. Quantitative parameters including tractogram volumes and number of fibers, which have already been used in previous studies [30,31], permitted to distinguish the mildest forms from other forms. Descriptive analyses of fiber bundles showed a preservation of ETP and an altered organization of intra- and interhemispheric connections in all patients, mostly with a loss of vertical and transversal connectivity and an apparent over-representation of longitudinal fibers. This suggests that tractography assessment is easy in mono-directional and partially myelinated fibers but remains difficult in multi-directional and less myelinated fibers in hypomyelinating disorders. Descriptive analyses also revealed an age-dependent evolution of the tractograms in the largest classicalintermediate group. For the youngest patients, the increasing size of tractograms may correspond to different stages of myelination. While healthy 2-year-old children have already achieved myelination except in subcortical fibers, severe supratentorial hypomyelination is the main feature in PLP1related disorders [10] where myelination can partially improve until 12 years of age [9]. For adult patients presenting severe cerebral atrophy on conventional MRI, the decreased size of tractograms may reflect WM atrophy or loss of connectivity. Laukka et al., argued prominent WM atrophy in PMD/SPG2 patients [11], whereas we observed parallel WM/GM atrophy over time on conventional MRI [9]. Taken together, the combination of patients' clinical evolution, progressive global atrophy, 
GM microstructure changes on DTI and progressive WM fiber disappearance on tractography converge to confirm that PLP1-related disorders are global CNS diseases affecting both WM and GM.

This study has several limitations. First, recent studies suggested the potential for seemingly minor alterations in data acquisition, in particular the b-value and number of diffusion gradient directions, to impact the DTI parameters [32]. Moreover, using multiple b-values such as recent technique called neurite orientation dispersion and density imaging (NODDI) could provide additional information on tissue compartments [33]. Further studies should consider these parameters. Second, with only 6 gradient directions, tractography can be limited to only major fiber tracts and can underestimate the connectivity. Our goal to differentiate severity in PLP1-related disorders was achieved in this protocol but it would be interesting to increase the number of directions to study weak connections. Finally, even if we took into account the age of the subjects as a covariate, there is a difference of age between the patients' groups that could generate a bias of interpretation. We did not report intraindividual evolution of DTI parameters and tractography. This would be a different study that could help to strengthen our results.

In conclusion, this study shows for the first time that tractography can be a useful and non-invasive technique in investigations and follow-up of hypomyelinating disorders. Our findings also bring further evidence of physiopathological mechanisms in PLPI-related disorders that likely involve both grey and white matter and infratentorial structures. These findings must be confirmed in further studies and may be important in helping to develop new therapeutic strategies targeting not only oligodendrocytes but also axons and neurons. 


\section{Acknowledgements}

The authors thank the members of the Leukofrance network who send us the patients (MA BarthezCarpentier, F Boidin, P Burkart, S Ceylaner, B Chabrol, D Chaigne, G Cioni, B Echenne, M Elleder, P Evrard, M Garcia Silva, K Kluger, J M Lopez-Terradas, J Motte, A Munnich, D Nicholls, N Philip, G Ponsot, T Reckert, D Rodriguez, C Rousselle, G Sébire, H Steinbock, M Troncoso, H Van Esche, and L Van Maldergem) and Dr JP Renou for precious help with the DTI analyses.

\section{Funding}

This study received funding from the ELA foundation, the FP7-RD Connect, the national PHRC [hospital-based clinical research program] for Clermont-Ferrand university hospital, and the Région Auvergne regional council.

JJL, CS, AS, JC, NS report a team non-financial support from BrainLab. The other authors declare no competing interests.

\section{List of Supplementary Material}

- Supplementary Material 1. Details of acquisition of T1-weighted, T2-weighted and FLAIR sequences.

- Supplementary Material 2. List of ROI studied for quantitative DTI.

- Supplementary Table 1. Results of statistical analyses for quantitative DTI parameters in white and grey matter.

- Supplementary Table 2. Results of statistical correlation between GMFM and quantitative DTI parameters in white and grey matter.

- Supplementary Table 3. Results of statistical analysis for quantitative parameters of left and right tractograms in each group of patients.

- Supplementary Figure 1. Additional results of statistical analyses for the different biomarkers: representation of effect sizes.

- Supplementary Figure 2. Superior views of both hemisphere tractograms of different severity groups and ages in PMD/SPG2 patients.

- Supplementary Figure 3. Lateral left views of right hemisphere tractogram of different severity groups and ages in PMD/SPG2 patients. 


\section{References}

1. Gow A, Gragerov A, Gard A, Colman DR, Lazzarini RA: Conservation of topology, but not conformation, of the proteolipid proteins of the myelin sheath. J Neurosci 1997;17:181-189.

2. Klugmann M, Schwab MH, Pühlhofer A, Schneider A, Zimmermann F, Griffiths IR, Nave KA: Assembly of CNS myelin in the absence of proteolipid protein. Neuron 1997;18:59-70.

3. Cailloux F, Gauthier-Barichard F, Mimault C, Isabelle V, Courtois V, Giraud G, Dastugue B, Boespflug-Tanguy O: Genotype-phenotype correlation in inherited brain myelination defects due to proteolipid protein gene mutations. Clinical European Network on Brain Dysmyelinating Disease. Europ J Hum Genet 2000;8:837-845.

4. Inoue K: PLP1-related inherited dysmyelinating disorders: Pelizaeus-Merzbacher disease and spastic paraplegia type 2. Neurogenetics 2005;6:1-16.

5. Nave KA, Bloom FE, Milner RJ: A single nucleotide difference in the gene for myelin proteolipid protein defines the jimpy mutation in mouse. J Neurochem 1987;49:1873-1877.

6. Griffiths IR, Scott I, McCulloch MC, Barrie JA, McPhilemy K, Cattanach BM: Rumpshaker mouse: new X-linked mutation affecting myelination: evidence for a defect in PLP expression. J Neurocytol 1990;19:273-283.

7. Garbern JY, Cambi F, Tang XM, Sima AA, Vallat JM, Bosch EP, Lewis R, Shy M, Sohi J, Kraft G, Chen KL, Joshi I, Leonard DG, Johnson W, Raskind W, Dlouhy SR, Pratt V, Hodes ME, Bird T, Kamholz J: Proteolipid protein is necessary in peripheral as well as central myelin. Neuron 1997;19:205-218.

8. Boespflug-Tanguy O, Labauge P, Fogli A, Vaurs-Barriere C: Genes involved in leukodystrophies: a glance at glial functions. Curr Neurol Neurosci Rep 2008;8:217-229.

9. Sarret C, Lemaire JJ, Tonduti D, Sontheimer A, Coste J, Pereira B, Feschet F, Roche B, Boespflug-Tanguy O: Time-course of myelination and atrophy on cerebral imaging in 35 patients with PLP1-related disorders. Dev Med Child Neurol 2016;58:706-713.

10. Schiffmann R, Boespflug-Tanguy O: An update on the leukodsytrophies. Curr Opin Neurol 2001;14:789-794.

11. Laukka JJ, Stanley JA, Garbern JY, Trepanier A, Hobson G, Lafleur T, Gow A, Kamholz J: Neuroradiologic correlates of clinical disability and progression in the X-linked leukodystrophy Pelizaeus-Merzbacher disease. J Neurol Sci 2013;335:75-81.

12. Sumida K, Inoue K, Takanashi J, Sasaki M, Watanabe K, Suzuki M, Kurahashi H, Omata T, Tanaka M, Yokochi K, Iio J, Iyoda K, Kurokawa T, Matsuo M, Sato T, Iwaki A, Osaka H, Kurosawa K, Yamamoto T, Matsumoto N, Maikusa N, Matsuda H, Sato N: The magnetic 
resonance imaging spectrum of Pelizaeus-Merzbacher disease: A multicenter study of 19 patients. Brain Dev 2016;38:571-580.

13. Laukka JJ, Makki MI, Lafleur T, Stanley J, Kamholz J, Garbern JY: Diffusion tensor imaging of patients with proteolipid protein 1 gene mutations. J Neurosci Res 2014;92: 1723-1732.

14. Russell DJ, Rosenbaum PL, Cadman DT, Gowland C, Hardy S, Jarvis S: The gross motor function measure: a means to evaluate the effects of physical therapy. Dev Med Child Neurol 1989;31:341-352.

15. Basser PJ, Mattiello J, LeBihan D. MR diffusion tensor spectroscopy and imaging. Biophys J 1994;66:259-267.

16. Mori S, van Zijl PC: Fiber tracking: principles and strategies - A technical review. NMR Biomed 2002;15:468-480.

17. Lazar M, Weinstein DM, Tsuruda JS, Hasan KM, Arfanakis K, Meyerand ME, Badie B, Rowley HA, Haughton V, Field A, Alexander AL: White matter tractography using diffusion tensor deflection. Hum Brain Mapp 2003;18:306-321.

18. Rothman KJ: No adjustments are needed for multiple comparisons. Epidemiology 1990;1:43-46.

19. Feise RJ: Do multiple outcome measures require p-value adjustment? BMC Med Res Methodol 2002;2:8.

20. Cohen J: Statistical power analysis for the behavioral sciences, ed 2. New Jersey, Lawrence Erlbaum, 1988

21. Steenweg ME, Vanderver A, Blaser S, Bizzi A, de Koning TJ, Mancini GM, van Wieringen WN, Barkhof F, Wolf NI, van der Knaap MS: Magnetic resonance imaging pattern recognition in hypomyelinating disorders. Brain 2010;133:2971-2982.

22. Hassen WB, Bégou M, Traore A, Moussa AB, Boehm N, Ghandour MS, Renou JP, Boespflug-Tanguy O, Bonny JM: Characterisation of spinal cord in a mouse model of spastic paraplegia related to abnormal axono-myelin interactios by in vivo quantitative MRI. Neuroimage 2009;46:1-9.

23. Sima AA, Pierson CR, Woltjer RL, Hobson GM, Golden JA, Kupsky WJ, Schauer GM, Bird TD, Skoff RP, Garbern JY: Neuronal loss in Pelizaeus-Merzbacher disease differs in various mutations of the proteolipid protein 1. Acta Neuropathol 2009;118:531-539.

24. Cavallari M, Ceccarelli A, Wang GY, Moscufo N, Hannoun S, Matulis CR, Jackson JS, Glanz BI, Bakshi R, Neema M, Guttmann CR: Microstructural changes in the striatum and 
their impact on motor and neuropsychological performance in patients with multiple sclerosis. PLoS One 2014;9:e101199.

25. Calabrese M, Rinaldi F, Seppi D, Favaretto A, Squarcina L, Mattisi I, Perini P, Bertoldo A, Gallo P: Cortical diffusion-tensor imaging abnormalities in multiple sclerosis: a 3-year longitudinal study. Radiology 2011;61:891-898.

26. Bradbury A, Peterson D, Vite C, Chen S, Ellinwood NM, Provenzale J: Diffusion tensor imaging analysis of the brain in the canine model of Krabbe disease. Neuroradiol $\mathbf{J}$ 2016;29:417-424.

27. Miller MJ, Haxhiu MA, Georgiadis P, Gudz TI, Kangas CD, Macklin WB: Proteolipid protein gene mutation induces altered ventilatory response to hypoxia in the myelin-deficient rat. J Neurosci 2003;23:2265-2273.

28. Bongarzone ER, Campagnoni CW, Kampf K, Jacobs EC, Handley VW, Schonmann V, Campagnoni AT: Identification of a new exon in the myelin proteolipid protein gene encoding novel protein isoforms that are restricted to the somata of oligodendrocytes and neurons. J Neurosci 1999;19:8349-8357.

29. Sarret C, Combes P, Micheau P, Gelot A, Boespflug-Tanguy O, Vaurs-Barriere C: Novel neuronal proteolipid protein isoforms encoded by the human myelin proteolipid protein 1 gene. Neurosci 2010;166:522-538.

30. Vassal F, Schneider F, Boutet C, Jean B, Sontheimer A, Lemaire JJ: Combined DTI tractography and functional MRI study of the language connectome in healthy volunteers: extensive mapping of white matter fascicles and cortical activations. PLoS One 2016;11:e0152614.

31. Wang PS, Yeh CL, Lu CF, Wu HM, Soong BW, Wu YT: The involvement of supratentorial white matter in multiple system atrophy: a diffusion tensor imaging tractography study. Acta Neurol Belg 2017;117:213-220.

32. Bisdas S, Bohning DE, Besenski N, Nicholas JS, Rumboldt Z: Reproducibility, interrater agreement, and age-related changes of fractional anisotropy measures at 3T in healthy subjects: effect of the applied b-value. AJNR Am J Neuroradiol 2008;29:1128-1133.

33. Zhang H, Schneider T, Wheeler-Kingshott CA, Alexander DC: NODDI: practical in vivo neurite orientation dispersion and density imaging of the human brain. Neuroimage 2012;61:1000-1016. 
Table 1. List of patients and families with PMD/SPG2

\begin{tabular}{|c|c|c|c|c|c|c|}
\hline Patient & Family & MDS & $\begin{array}{c}\text { Age at DTI } \\
\text { acquisition } \\
\text { +/- GMFM } \\
\text { (years) }\end{array}$ & $\begin{array}{l}\text { GFMF88 at } \\
\text { MRI } \\
\text { acquisition }\end{array}$ & $\begin{array}{c}P L P 1 \\
\text { mutation }\end{array}$ & $\begin{array}{c}\text { Family } \\
\text { genetic } \\
\text { number* }\end{array}$ \\
\hline 1 & 1 & 2 & 2.3 & 29.3 & duplication & 1910 \\
\hline 2 & 2 & 0 & 3.0 & NA & c. $454-1 \mathrm{G}>\mathrm{A}$ & 767 \\
\hline 3 & 3 & 1 & 3.5 & 36.8 & c.327delC & 1171 \\
\hline 4 & 4 & 2 & 3.8 & 25.3 & duplication & NA \\
\hline 5 & 5 & 2 & 4.3 & 34.8 & duplication & 1251 \\
\hline 6 & 6 & 2 & 4.4 / 6.8 & $26 / 36.8$ & duplication & 1059 \\
\hline 7 & 7 & 2 & $4.5 / 7.5$ & NA / 39.5 & c. $659 \mathrm{G}>\mathrm{A}$ & 759 \\
\hline 8 & 8 & 2 & 4.7 & NA & duplication & 1201 \\
\hline 9 & 9 & 2 & 5.1 & 38.4 & duplication & 1026 \\
\hline 10 & 10 & 3 & 5.5 & 39 & duplication & 1114 \\
\hline 11 & 11 & 1 & 5.7 & 28.7 & duplication & 968 \\
\hline 12 & 12 & 2 & 5.7 & 26.7 & duplication & 887 \\
\hline 13 & 13 & 2 & 6.2 & 47.1 & c.125delG & 1038 \\
\hline 14 & 14 & 3 & $8.3 / 11.5$ & 44.2 / NA & duplication & 407 \\
\hline 15 & 15 & 0 & 8.5 & 20.5 & duplication & 545 \\
\hline 16 & 16 & 1 & 8.6 & 17 & duplication & 1776 \\
\hline 17 & 17 & 0 & 8.6 & NA & c. $670 \mathrm{C}>\mathrm{T}$ & 766 \\
\hline 18 & 18 & 2 & 8.6 & 41.4 & duplication & 418 \\
\hline 19 & 19 & 3 & $10.8 / 13.3$ & NA / 45 & c. $173 A>G$ & 106 \\
\hline 20 & 20 & 1 & 12.3 & NA & duplication & 440 \\
\hline 21 & 21 & 3 & 13.5 & NA & duplication & 46 \\
\hline 22 & 22 & 2 & 13.6 & 46.9 & duplication & 154 \\
\hline 23 & 23 & 3 & $14.5 / 17.4$ & 49.9 / 30.5 & c.307delG & 905 \\
\hline 24 & 24 & 2 & 14.8 & 40 & duplication & 61 \\
\hline 25 & 25 & 1 & 15.0 & NA & duplication & 150 \\
\hline 26 & 26 & 4 & 16.2 & 56.6 & c. $548 \mathrm{C}>\mathrm{A}$ & 41 \\
\hline 27 & 27 & 2 & $16.8 / 19.1$ & $0 / 0$ & c. $608 \mathrm{~A}>\mathrm{G}$ & 37 \\
\hline 28 & 28 & 2 & 17.0 & 24 & duplication & 28 \\
\hline 29 & 23 & 3 & 17.2 / 20.1 & 44.4 / 32.3 & c.307delG & 905 \\
\hline 30 & 29 & 0 & 17.4 & NA & c. $454-1 G>T$ & 26 \\
\hline 31 & 30 & 3 & 19.0 & 24 & c. $646 \mathrm{C}>\mathrm{G}$ & 53 \\
\hline 32 & 31 & 2 & 21.8 & NA & c. $646 \mathrm{C}>\mathrm{T}$ & 24 \\
\hline 33 & 19 & 3 & 32.8 & NA & c. $173 A>G$ & 106 \\
\hline 34 & 32 & 4 & $37.0 / 41.0$ & 31.8 & c. $801 \mathrm{G}>\mathrm{T}$ & 1 \\
\hline 35 & 7 & 2 & 43.5 & NA & c. $659 \mathrm{G}>\mathrm{A}$ & 759 \\
\hline
\end{tabular}

MDS: motor developmental score, age (years) at DTI acquisition and GMFM scoring, GMFM: gross motor function measure realized at the time of MRI acquisition, and genotype (at the DNA level). * Family genetic number corresponds to our laboratory family number and refers to previous publication (Cailloux et al. 2000). NA : not available. 


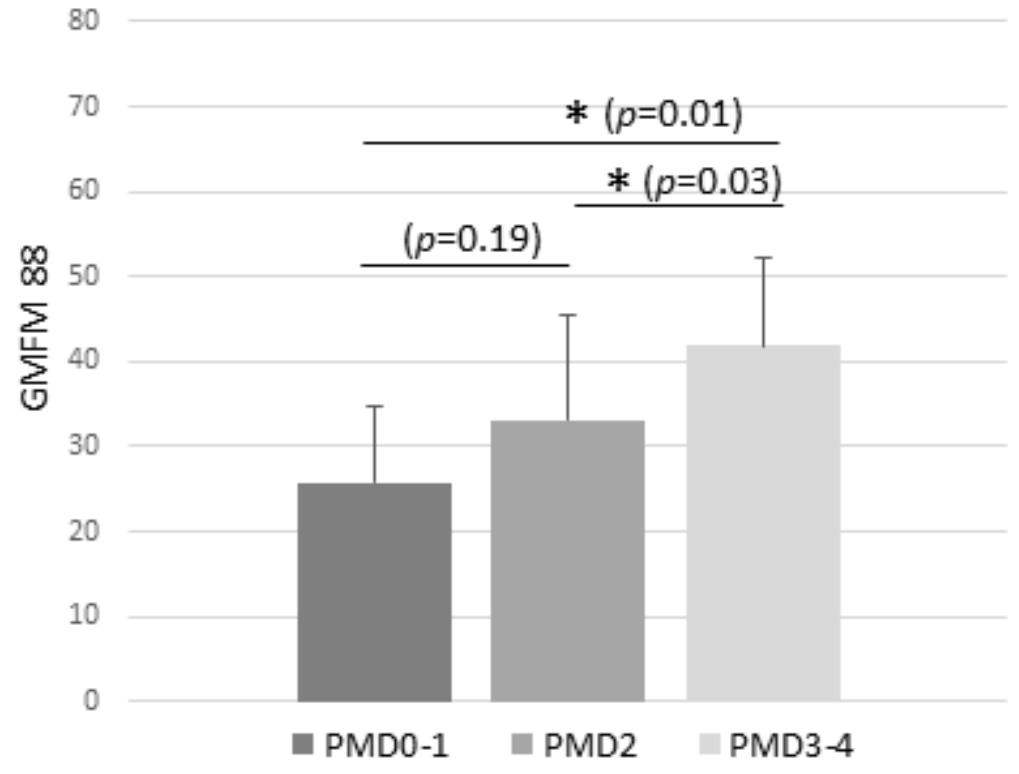

Figure 1. Comparison of mean GMFM between the three MDS groups. 

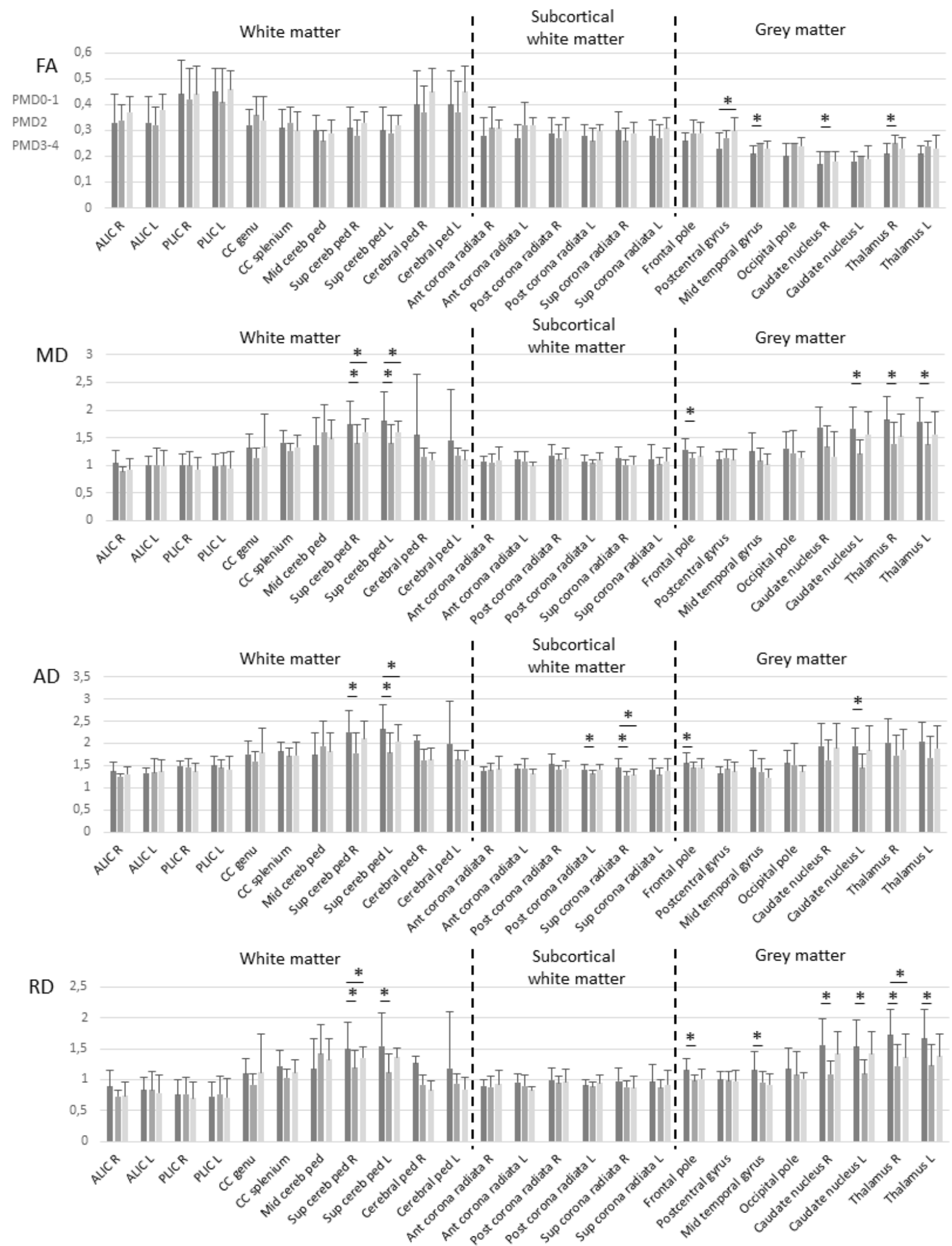

Figure 2. Comparison of quantitative DTI parameters between the three MDS groups in white matter, subcortical white matter and grey matter. 


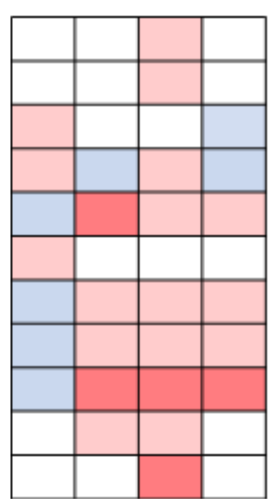

FA MD AD RD

White matter

ALIC R

ALIC L

PLIC R

PLICL

$-1$

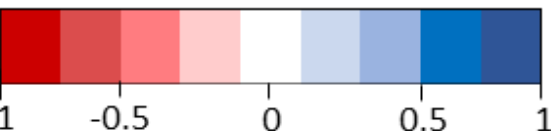

CC genu

CC splenium Mid cereb ped Sup cereb ped $R$ Sup cereb ped $L$ Cerebral Ped R Cerebral Ped L

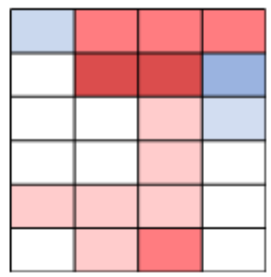

FA $\quad M D \quad A D \quad R D$

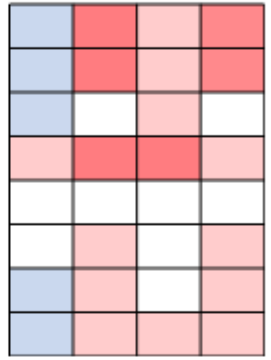

Subcortical white matter Sup corona radiata L

Ant corona radiata $\mathrm{R}$ Ant corona radiata L Post corona radiata $\mathrm{R}$ Post corona radiata $L$ Sup corona radiata $\mathrm{R}$

FA MD AD RD

Grey matter
Frontal pole Occipital pole Post-central gyrus Mid temporal gyrus Caudate nucleus $\mathrm{R}$ Caudate nucleus $L$ Thalamus $\mathrm{R}$ Thalamus L

Figure 3. Coefficients of correlation between GMFM and quantitative DTI parameters in white and grey matter.

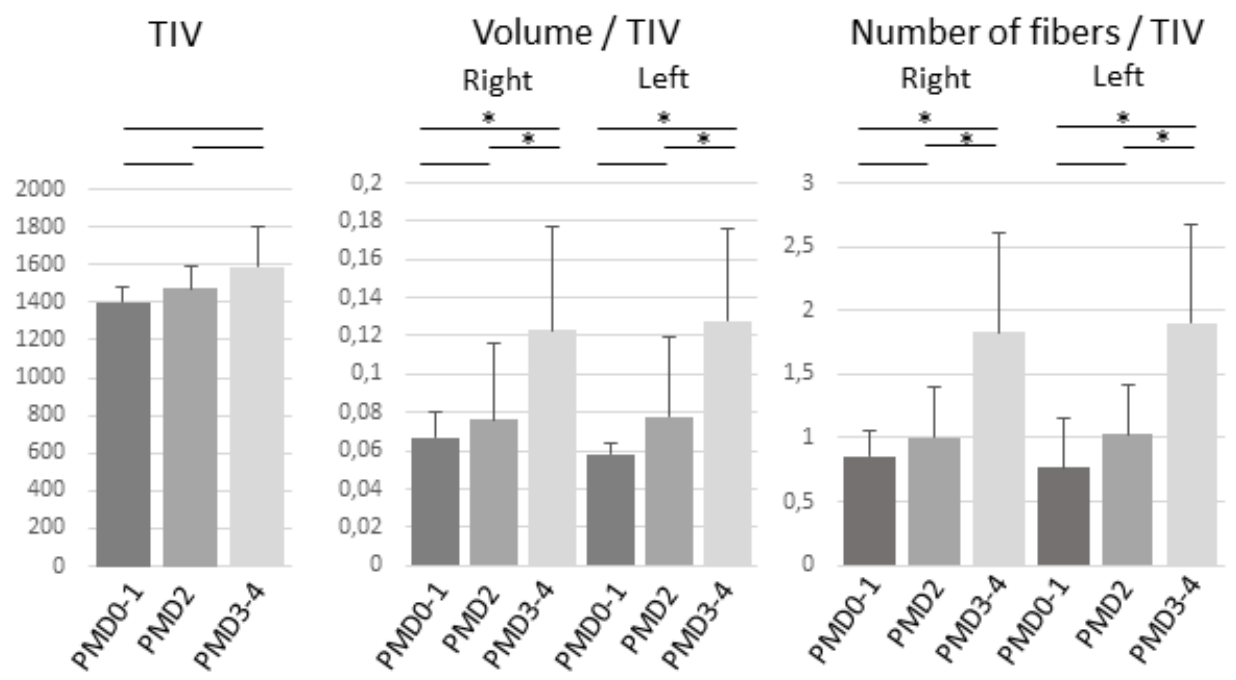

Figure 4. Comparison of quantitative tractography parameters between the three MDS groups. 
A.

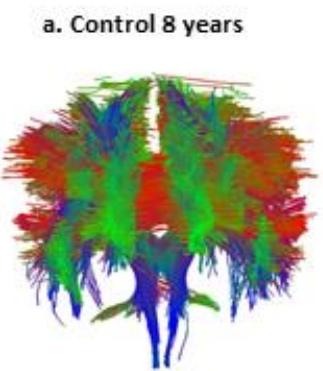

e. Control adult

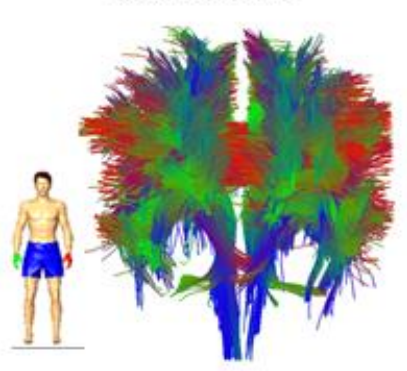

b. PMD0 8.5 years GMFM $=20.5$

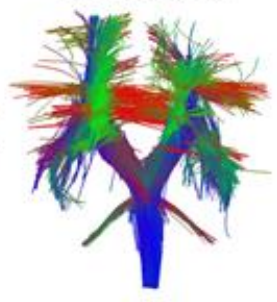

f. PMD0 17.4 years GMFM not available

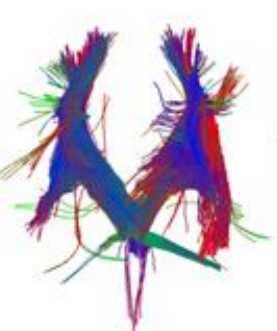

c. PMD2 8.6 years GMFM $=41.4$

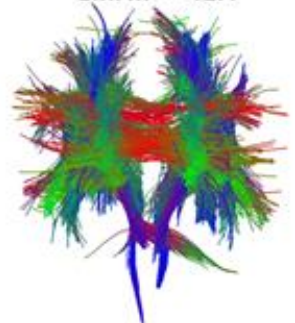

g. PMD2 17.0 years GMFM $=24$

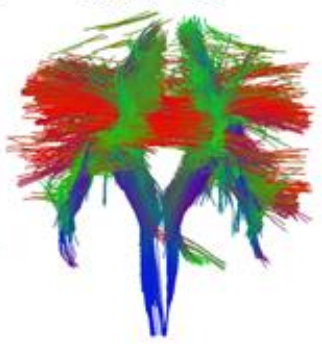

d. PMD3 10.8 years

GMFM $=45$

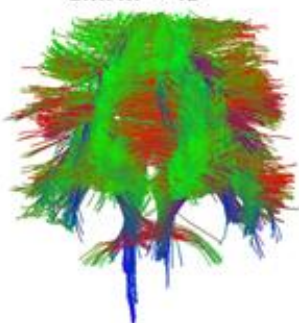

h. PMD4 16.2 years GMFM $=56.6$

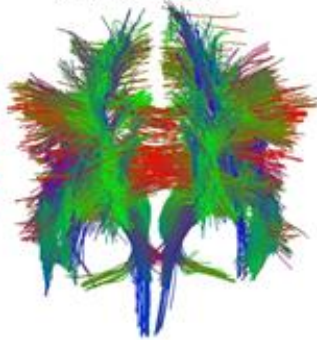

\begin{tabular}{|c|c|c|c|c|c|c|c|c|c|c|c|c|c|}
\hline \multirow{3}{*}{$\begin{array}{l}\text { Type } \\
\text { size } \\
\text { DMNS } \\
\end{array}$} & \multicolumn{3}{|c|}{ ETP } & \multicolumn{3}{|c|}{ CF } & \multicolumn{7}{|c|}{ IHC } \\
\hline & \multirow[t]{2}{*}{ large } & \multicolumn{2}{|c|}{ small lackling } & \multirow[t]{2}{*}{ large } & \multirow{2}{*}{\multicolumn{2}{|c|}{ small lacking }} & & large & & & small & & lacking \\
\hline & & & & & & & \multicolumn{7}{|c|}{ longitudinal vertical transversal longitudinal vertical transversal } \\
\hline PMD0-1 & 1 & 2 & - & 1 & 1 & 1 & - & - & - & 1 & - & - & 2 \\
\hline PMD2 & 11 & 1 & - & 4 & 6 & 2 & 2 & - & 1 & 7 & - & - & 2 \\
\hline PMD3.4 & 9 & - & - & 4 & 1 & 4 & 8 & - & - & 1 & - & - & - \\
\hline
\end{tabular}

B.

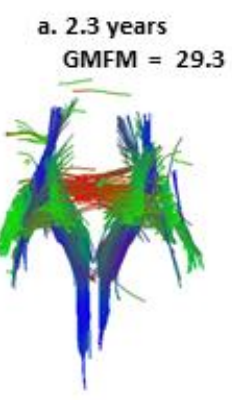

e. 13.6 years GMFM $=46.9$

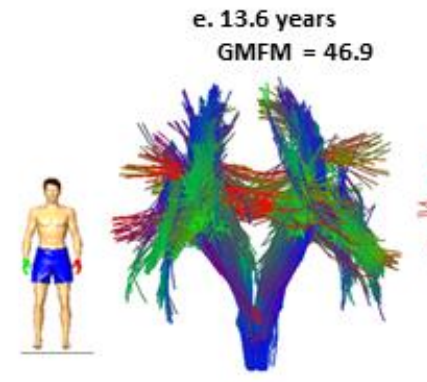

b. 4.5 years

GMFM not available

c. 6.8 years GMFM $=36.8$

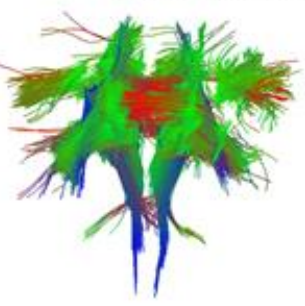

f. 17.0 years GMFM $=24$

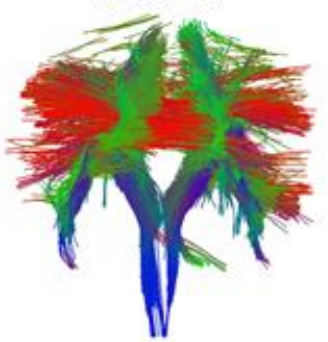

g. 21.8 years

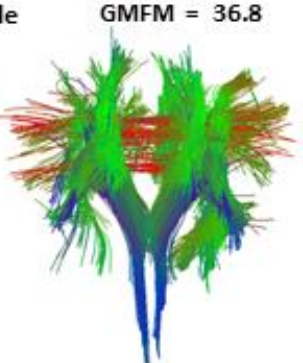
GMFM not available

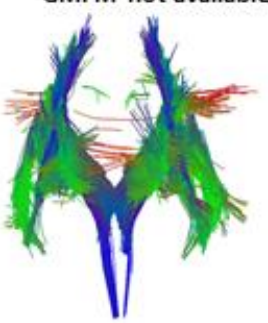

d. 8.6 years GMFM $=41.4$

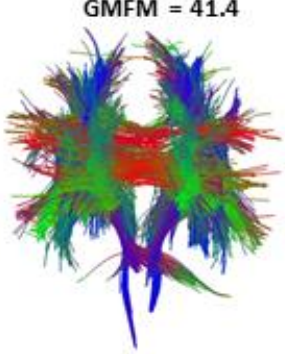

h. 43.5 years GMFM not available

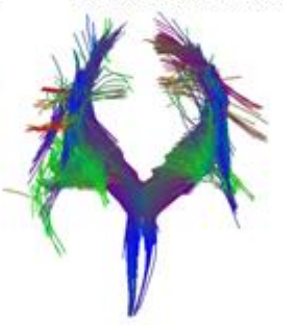

Figure 5. Examples of frontal views of both hemisphere tractograms and results of morphologic analyses of tractographies in different severity groups and ages in PMD/SPG2 patients. 


\section{Figure legends}

Figure 1. Comparison of mean GMFM between the three MDS groups. Y-axis: GMFM (Gross Motor Function Measure) includes 88 items (best motor score $=88$ ) at the time of MRI acquisition. $\mathbf{X}$-axis: MDS (Motor developmental score) corresponds to the best motor function achieved between the ages of 2 and 5 years. Thirty-two patients are divided in three groups of MDS: PMD0-1 (n=8), PMD2 ( $\mathrm{n}=14)$, PMD3-4 ( $\mathrm{n}=10)$. Results are given using ANCOVA with age as the adjustment covariate. $*$ indicates a significant difference $(p$-value $<0.05) . p=p$-value. Standard deviations are given as an error bar.

\section{Figure 2. Comparison of quantitative DTI parameters between the three MDS groups in white} matter, subcortical white matter and grey matter. Successive results for fractional anisotropy (FA), mean diffusivity (MD), axial diffusivity (AD) and radial diffusivity (RD). Thirty-two patients are divided in three groups of MDS: PMD0-1 ( $n=8)$, PMD2 ( $n=14)$, PMD3-4 ( $n=10)$. MD, AD and $\mathrm{RD}$ values are expressed as $10^{-3}$ values. Results are expressed as means. Standard deviations are given as an error bar. * for $p$-value $<0.05$. $p$-values were computed using ANCOVA with age as the adjustment covariate. ALIC: anterior limb of the internal capsule, PLIC: posterior limb of the internal capsule, R: right, L: left, CC: corpus callosum, Mid: middle, Ant: anterior, Post: posterior, Sup: superior, Ped: peduncle, Cereb: cerebellar.

Figure 3. Coefficients of correlation between GMFM and quantitative DTI parameters in white matter, subcortical white matter and grey matter. Using a nonparametric Spearman's correlation test, with Sidak's type-I error correction, values of coefficients of correlation between -1 and 1 are represented graphically with a color-coded heatmap in each region of interest and for each DTI parameters. GMFM: gross motor function measure, FA: fractional anisotropy, MD: mean diffusivity, AD: axial diffusivity, RD: radial diffusivity, ALIC: anterior limb of internal capsule, PLIC: posterior limb of internal capsule, R: right, L: left, CC: corpus callosum, Mid: middle, Ant: anterior, Post: posterior, Sup: superior, Ped: peduncle, Cereb: cerebellar.

\section{Figure 4. Comparison of quantitative tractography parameters between the three MDS groups.} Successive results for total intracranial volume (TIV), right and left tractogram volume / TIV and right and left tractogram number of fibers / TIV. Twenty-four patients whom tractography was available are divided in three groups of MDS: PMD0-1 ( $n=3)$, PMD2 ( $=12)$, PMD3-4 (n=9). Results are expressed as means. Standard deviations are given as an error bar. Total intracranial 
volume (TIV) and tractogram volume are expressed in $\mathrm{mm}^{3}$. * for $p$-value $<0.05 . p$-values were computed using ANCOVA with age as the adjustment covariate.

Figure 5. Examples of frontal views of both hemisphere tractograms and morphological analyses of tractographies of different MDS groups and ages in PMD/SPG2 patients.

Tractograms were realized for two regions of interest corresponding to the right/left hemispheres. Colors correspond to the direction of main-stream fibers (DMSF): blue = vertical direction, green = longitudinal direction, red $=$ transversal direction. For clinical comparison, Gross Motor Functional Measure (GMFM) at the time of MRI is given under each tractogram when available. A. Examples of tractograms for each group of severity and table of the results of morphological analyses in 24 patients whom tractography was available: PMD0-1 ( $n=3)$, PMD2 ( $n=12)$ and PMD3-4 (n=9). Extratelencephalic projections (ETP) were relatively preserved in all groups, but were smaller than in controls. Compared to controls (a,e), commissural fibers (CF) and intrahemispheric connections (IHC) were smaller or lacking in all groups: (1) the most severe PMD0-1 (b,f), (2) the classical PMD2 (c,g) and the mildest PMD3-4 patients (d,h). DMSF in IHC was marked by longitudinal (or rare transversal) over representation. B. Each image corresponds to tractograms of PMD2 patients of different ages. These results show that (1) the youngest patients (a,b) had less-developed ETP, CF and IHC than patients at the late infancy (c,d) and adolescent patients (e,f); (2) the oldest patients (g,h) had smaller ETP, CF and IHC than adolescent patients (e,f). 